\title{
If not now, when? A clinical perspective on the unprecedented challenges facing ICUs during the COVID-19 pandemic
}

\author{
Ignacio Martin-Loeches ${ }^{1,2^{*}}$ (D) Yaseen Arabi $^{3}$ and Giuseppe Citerio ${ }^{4,5}$
}

(C) 2021 Springer-Verlag GmbH Germany, part of Springer Nature

Intensive care units (ICUs) worldwide continue to struggle with the massive influx of patients with critical illness associated to coronavirus disease 2019 (COVID19). Their capacity is overwhelmed and there is clearly a need to act to implement a crisis standard of care, in an attempt to mitigate disparities in access to intensive care. The process of characterizing the natural progression of a disease and the pathophysiological differences between different categories of critically ill patients requires many years of in-depth study. And even when such characterization is available to help us make clinical judgments, we, as intensivists, continue to face challenges in achieving consensus and implementing critical care guidelines. The COVID-19 pandemic has led to a complete change in the way we conduct research, interpret results, and make recommendations. Over the past year, immediacy has become the dominant theme, favored over quality.

One particularly important feature of the current emergency has been the change we are seeing in the type of patients being admitted to ICUs. At the start of the COVID-19 pandemic, reports indicated that the disease mostly affected older adults and that young people were more likely to have milder disease. At that time, we were warned that our main focus should be to reduce infection and subsequent transmission to persons at higher risk of severe illness. Over time, however, this global consensus started to change. According to data from epidemiological teams at the Centers for Disease Control and

\footnotetext{
*Correspondence: drmartinloeches@gmail.com

${ }^{1}$ Department of Intensive Care Medicine, St. James's University Hospital, Multidisciplinary Intensive Care Research Organization (MICRO), Trinity Centre for Health Sciences, James's Street, Dublin 8, P.O. Box 580, Dublin, Ireland

Full author information is available at the end of the article
}

Prevention in the United States, people under the age of 30 accounted for more than 20\% of COVID-19 cases over the summer of 2020 [1]. This trend was subsequently validated, as reported in a study from Brazil by Kurtz et al. [2], published in this issue of Intensive Care Medicine. This group of researchers conducted an observational study in over 13,000 patients and determined that progressively more younger patients were being admitted to ICUs in Brazil. Importantly, however, this study only analyzed patients admitted until October 2020, and it could therefore be argued that the age at admission has since decreased further, in line with the findings of a recent US report that observed severe clinical characteristics in 177 pediatric and young adult patients with SARS-CoV-2 infection, of whom 44 (25\%) required hospitalization. Of these, $20 \%$ were critically ill [8].

The main question now is: what led to this change in the age of patients being admitted to ICUs? We have several potential explanations. (1) Older patients being admitted to ICUs might have a poor outcome and this could have an impact on the admission criteria. Grasselli et al. reported mortality rates of $40 \%$ in the over- $70 \mathrm{~s}$ and of $>50 \%$ in the over- $80 \mathrm{~s}$ at the start of the pandemic in Italy [3]. (2) The new variants observed in SARS-CoV2-infected patients are undeniably having an impact. There is no doubt that the prominent strain in Brazil is now the Brazilian variant, which has been named P.1. Although it is not believed to be more deadly than the initially reported COVID-19 strain, P.1 is thought to be responsible for a rapid increase in the spread of infections. Moreover, a recent study has detailed a resurgence of COVID-19 in Manaus (Brazil), even though previous studies conducted in blood donors showed that $76 \%$

\section{Springer}


(95\% CI 67-98) of the population had been infected with SARS-CoV-2 by October 2020, a finding which suggested that pre-infection rates were above the theoretical herd immunity threshold (67\%) [4]. In view of these findings, studies to investigate genetic and immunological parameters and clinical and epidemiological characteristics of SARS-CoV-2 variants with SARS-CoV-2 lineages linked to clinical progression are surely warranted. (3) The number of younger patients being infected has grown. This hypothesis, however, has not been clinically validated on a large scale. It is important to stress that while more patients are younger, there has been no marked change in the age-related risk of becoming critically ill, as recently reported by different country surveillance reports [5].

A major trend noted during the pandemic is related to the increase in the use of non-invasive respiratory support (NIRS), including non-invasive mechanical ventilation or high-flow nasal cannula (HFNC) treatment. Whilst at the beginning of the pandemic, concerns about NIRS failure and aerosol transmission limited its use in hypoxemic patients admitted to ICUs, it is now common to see ICU patients being treated successfully with NIRS. This is a finding also reported in this issue of Intensive Care Medicine by our Brazilian colleagues [2], whose clinical report included patients admitted to 126 ICUs during an 8-month period. Their data show an increased use of NIRS as the first respiratory support measure, associated with high survival rates. Nonetheless, we must acknowledge the limitations that can arise due to the nature of such observational studies. NIRS has consistently been highlighted as a desirable element in clinical guidelines on the management of patients with severe hypoxemia. As intensivists, we try to implement 'crisis standards of care' while striving to minimize potential complications arising from invasive mechanical ventilation and COVID-19. Accordingly, due to limited ICU bed and resource availability, the utilization of these devices has increased in patients who are critically ill.

This finding also has important economic implications, as the NIRS market is expected to garner considerable growth ( $\sim 6$ percent during the assessment period of 2019-2027) on the back of the escalating medical emergencies and the rising need to manage critical patients around the world, as reported, by experts, in Transparency Market Research [6]. However, as the use of NIRS is increasing, clinicians should be alert to early signs of failure of NIRS applications in critical care. For example, some index criteria/guidelines, such as the severity of the disease and the ROX index, defined as the ratio of oxygen saturation as measured by pulse oximetry $/ \mathrm{FiO}$ to respiratory rate, have been assessed as predictors of the need to intubate COVID-19 patients receiving HFNC oxygen therapy [7].

Even though there have been many recent developments, and clinical reports underlining the ways in which COVID-19 patients have benefited from critical care management approaches and treatment changes, unfortunately the number of extremely sick patients being admitted to ICUs with acute respiratory distress syndrome (ARDS) and ultimately requiring invasive mechanical ventilation remains high. It is important to determine what factors are associated with disease progression, especially over the first days of invasive mechanical ventilation. This is supported by Patel et al. [8], who analyzed clinical data of 633 patients under invasive mechanical ventilation in the UK and noted an extremely high mortality rate (43.3\%), rising to over $80 \%$ in 3 of the 13 participating centers. Increased age was associated with worsening of hypoxemia within the first 7 days. In this manuscript, the authors implemented a machine learning and explainable artificial intelligence tool to predict accuracy for mortality compared with admission data. Surprisingly, prone positioning, which has been one of the most life-saving maneuvers implemented during the COVID-19 pandemic, was applied in only one in four patients with moderate hypoxemia, and in less than half of those with severe hypoxemia. There is a definite need for improvements, but in saying that, it must also be pointed out that the UK and Ireland are among the countries with the lowest ratio of ICU beds per population in Europe [9], which directly impacts the ability to provide immediate, lifesaving and quality care.

The COVID-19 pandemic is an unprecedented medical and economic challenge for intensivists, who face a relative scarcity of critical resources, and for critically ill patients in need of therapeutic strategies available only in ICUs. In the absence of robust and sustained governmental support, almost all ICUs will continue to face sizeable challenges in delivering quality care to those most at risk. There are many take-home messages, but one of the most crucial is this: there is clearly a need to invest in intensive care medicine, and if not now, when?

\footnotetext{
Author details

${ }^{1}$ Department of Intensive Care Medicine, St. James's University Hospital, Multidisciplinary Intensive Care Research Organization (MICRO), Trinity Centre for Health Sciences, James's Street, Dublin 8, P.O. Box 580, Dublin, Ireland. ${ }^{2}$ Hospital Clinic, IDIBAPS, Universidad de Barcelona, CIBERes, Barcelona, Spain. ${ }^{3}$ Intensive Care Department, King Abdullah International Medical Research Center, Ministry of National Guard Health Affairs, King Saud Bin Abdulaziz University for Health Sciences, Riyadh, Kingdom of Saudi Arabia. ${ }^{4}$ School of Medicine and Surgery, University of Milano-Bicocca, Monza, Italy. ${ }^{5}$ Neurointensive Care Unit, San Gerardo Hospital, ASST-Monza, Monza, Italy.
} 


\section{Declarations}

\section{Conflict of interest}

The authors declare that they have no conflicts of interest.

\section{Publisher's Note}

Springer Nature remains neutral with regard to jurisdictional claims in published maps and institutional affiliations.

Received: 31 March 2021 Accepted: 3 April 2021

Published online: 11 May 2021

\section{References}

1. Boehmer TK, DeVies J, Caruso E et al (2020) Changing age distribution of the COVID-19 pandemic - nited States, May-August 2020. MMWR Morb Mortal Wkly Rep 69:1404-1409. https://doi.org/10.15585/mmwr.mm693 9e1

2. Kurtz et al (2021) Evolving changes in mortality of 13,301 critically ill adult patients with COVID-19 over 8 months. Intensive Care Med. https://doi. org/10.1007/s00134-021-06388-0
3. Grasselli G, Zangrillo A, Zanella A et al (2020) Baseline characteristics and outcomes of 1591 patients infected with SARS-CoV-2 admitted to ICUs of the Lombardy Region, Italy. JAMA J Am Med Assoc 323:1574-1581. https://doi.org/10.1001/jama.2020.5394

4. Sabino EC, Buss LF, Carvalho MPS et al (2021) Resurgence of COVID-19 in Manaus, Brazil, despite high seroprevalence. Lancet 397:452-455

5. https://www.hpsc.ie/a-z/respiratory/coronavirus/novelcoronavirus/surve illance/epidemiologyofcovid-19inirelandweeklyreports/COVID-19\% 20Weekly\%20Report_\%20Week\%202_\%20Slidset_HPSC\%20-\%20Web site.pdf

6. https://www.researchandmarkets.com/reports/4851376/non-invasiveventilators-market-global-industry\#pos-0

7. Mellado-Artigas R, Mujica LE, Ruiz ML et al (2021) Predictors of failure with high-flow nasal oxygen therapy in COVID-19 patients with acute respiratory failure: a multicenter observational study. J Intensive Care 9:23. https://doi.org/10.1186/s40560-021-00538-8

8. Patel et al (2021) Natural history, trajectory, and management of mechanically ventilated COVID-19 patients in the United Kingdom. Intensive Care Med.https://doi.org/10.1007/s00134-021-06389-z

9. Rhodes A, Ferdinande P, Flaatten $\mathrm{H}$ et al (2012) The variability of critical care bed numbers in Europe. Intensive Care Med 38:1647-1653. https:// doi.org/10.1007/s00134-012-2627-8 